\title{
KNOWLEDGE MANAGEMENT AND ORGANISATIONAL EFFECTIVENESS: BALANCING THE MILD, THE WILD, AND THE CRAZY
}

\author{
Greg Marsh \\ Defence Science \& Technology Organisation \\ PO Box 1500 Edinburgh, South Australia, 5111 \\ Phone: 0882595362 \\ Fax: $\quad 0882595619$ \\ Email: greg.marsh@dsto.defence.gov,au \\ Martin Burke \\ Defence Science \& Technology Organisation \\ and Systems Engineering and Evaluation Centre, University of South Australia \\ PO Box 1500 Edinburgh, South Australia, 5111 \\ Phone: 0882597004 \\ Fax: $\quad 0882595619$ \\ Email: $\quad$ martin.burke@dsto.defence.gov.au
}

\begin{abstract}
In the Knowledge Age, effective Knowledge Management (KM) is seen as fundamental to the survival of many organisations. Information Systems (IS) increasingly underpin KM in such organisations. The value of the contribution of IS to the goals of these organisations depends on balancing various conflicting requircments. For cxample, stable, robust and reliable business processes are often fundamental to their efficient operation. However, less structured environments are conducive to the innovation and knowledge creation that are considered to be fundamental to their on-going effective operation. The paper presents a model developed to relate these conflicting requirements to organisational effectiveness and describes how it was used to examine the outcomes of a Knowledge Management study conducted in the Australian Defence Organisation.
\end{abstract}

\section{INTRODUCTION}

It has been observed (eg The Price Waterhouse LLP Change Integration Team 1996; Shaw 1997; Turner and Crawford 1998; Hidding 2001), that change is the imperative of the Knowledge Age. In this epoch, both public and private organisations operate in environments of constant, and often unpredictable, change.

This paper contends that, as the dynamics of environmental change have become more chaotic, traditional methods for its analysis and prediction have become less effective. Accordingly, conventional approaches of organisational response to change have lost their relevance. It argues that, in order to survive and thrive in such dynamic and uncertain circumstances, organisations need to develop new ways of balancing stability and dynamism in their functions, processes, and operations.

The paper presents a new model that addresses organisations interacting with external environments subject to dynamic change. The model applies insights from the fields of Complexity Science, Systems Thinking and Knowledge Management to treat organisations as systems comprising inter-related heterogenous components described in terms of attributes and relationships.

The paper describes the use of the model in attempting to resolve a paradox that emerged in a recent investigation of Knowledge Management in the Australian Defence Organisation.

\section{PARADIGM SHIFT IN UNDERSTANDING}

Some management writers (Freedman 1992; Stacey 1996) see developments in the science of complexity as providing a new paradigm for understanding organisations. The concept of the "paradigm" in scientific research was used by Thomas Kuhn (1970) to describe a set of unquestioned assumptions held by those who, at a particular point in time, profess a particular science2. Revolutionary changes in the dominant scientific paradigm profoundly influence how society views the world. "The historian of science may be tempted to exclaim that when paradigms change, the world itself changes with them"(Kuhn 1970). It can be said that our view of the business world has changed as we move from the Industrial Age to the Knowledge Age.

The success of Newtonian mechanics contributed to a socially constructed phenomenon in which science was seen as "exact" and the presumption that once all fundamental laws had been discovered, the future behaviour of systems could be predicted with certainty. In the 19th century, many scientists (notably Laplace) were confident that even the

\footnotetext{
2 Usage of the term "paradigm" has changed over time to include the prevailing modes of thinking as well as the set of unquestioned assumptions.
} 
most complex behaviours could be reduced to simple laws, which could be used to predict future behaviour exactly. This conviction spilled over into other areas of thinking. For example, F.W. Taylor's (1923) analysis of organisations and his prescriptions for "scientific management" held the implicit assumption that once the fundamentals of any task were discovered, the one best way of performing it could be calculated (Pugh and Hickson 1996).

"But during the past few decades, more and more scientists have concluded that this and many other of science's traditional assumptions about the way nature works are fundamentally wrong. Far from being as predictable as clockwork, nature appears as random as a throw of the dice"(Freedman 1992, p 30). Physics led the way as quantum mechanics and relativity theories showed that the Newtonian paradigm was limited and, in the limit, that physical parameters could not be measured exactly. More recently, the mathematics of chaos theory has shown that even when the causal relationship is known, some systems exhibit dramatic and unpredictable outcomes in response to very small changes in initial conditions. It has been observed that complex systems, like the weather for example, are sensitive to initial conditions and can develop dramatically different behaviour from very small changes to initial conditions. This is known as the butterfly effect - the notion that a butterfly stirring the air today in Peking can transform the storm systems next month in New York (Gleik 1988).

Contemporary organisations have to operate in a rapidly changing environment, dealing with new and unexpected forms of competition, and working in new forms of partnerships and alliances. Corporations no longer only compete with traditional, similar organisations, but are subject to competition from unexpected quarters. For example IBM, accustomed to competing in a relatively stable mainframe market, had to change rapidly its strategy and processes to cope with the totally different competitive environment arising from the advent of the PC and MicroSoft's business strategies. Microsoft in turn has had to adapt its strategy very rapidly, to counter a new form of competition from Netscape (Rebello 1996).

It would seem that changes of thinking are occurring in other fields of study, including organisational and management science, that are analogous to the complexity/uncertainty paradigm shift in the physical sciences. The recognition of such limitations of organisation and management theory is leading to a new paradigm that acknowledges the reality of operating with complexity/uncertainty. Strategic planning for example, was developed in the 1960s with the implied assumption that the future was predictable, and only a few years ago Mintzberg reported that "Strategic planning, as it is still practised, is still heavily slanted toward the 'predict-and-prepare' mode of coping with the future" (Mintzberg 1994, p228). In the new paradigm of uncertainty, organisations are positioning themselves to meet likely contingencies and developing the flexibility to respond quickly to the unexpected threat or opportunity. The ability to manage the unpredictable is the objective of this repositioning.

"In the new thinking, success is defined not as ending up where you want to be, but as wanting to be where you end up"'(Sauer and Yetton 1997).

Figure 1 shows how the complexity/uncertainty analogy can be used to draw some insights about the interactions within an organisation and with its environment. Conventional relationships are in this "mild" region close to agreement and close to certainty. In this region we can be confident of causal relationship and can have high confidence of predicting future outcomes from the measured inputs.

Moving further out into the "wild" region, there is more complexity. Here there is some control, but the unexpected is likely. This is the area of creativity, new ideas, insights and innovation. As we move further out the ride becomes wilder as we move towards the edge of chaos. Here creativity and innovation is at its greatest, but levels of control and efficiency are relatively low. In the "crazy" region of highest uncertainty and greatest disagreement, chaos reigns. Here we expect confusion and disarray. The good ideas from here have to be pulled back towards the controlled region to gain the benefits. 


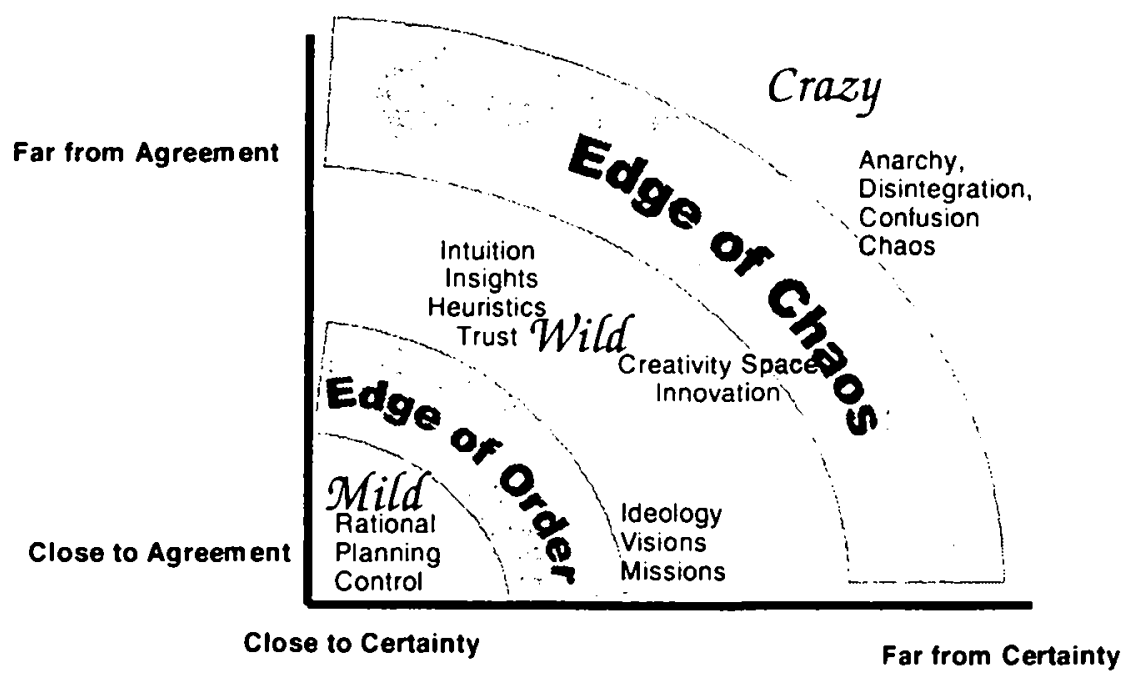

Based on: Ralph Stacey 1997

Figure 1. Mild, wild, and Crazy

\section{KNOWLEDGE IN ORGANISATIONS}

As society moves from the Industrial Age to the Knowledge Age, the predominant competitive mode is moving from efficiency in process to effectiveness in innovation and flexibility. Success of organisations depends not just on efficient management of physical and information resources but increasingly on the effective management of knowledge. This raises issues of the nature of the knowledge within organisations; how new knowledge is created and embodied in the innovations, and how existing knowledge might to be brought together either physically or virtually. In practice, the terms "data", "information" and "knowledge" are often confused (Boisot 1998). Data and information can be transmitted directly, but knowledge is "...anchored on the commitment and beliefs of its holder" Nonaka $(1994, \mathrm{p} 15)$.

It is the interaction between the information and the existing knowledge of the agent that is defining in this case. Data becomes information when the agent receiving it can ascribe meaning to the data; knowledge is generated through the interaction of existing knowledge with the flow of information to the agent. In contrast to information, knowledge cannot be directly observed. Its existence can only be inferred from the action of agents (Boisot 1998 p12).

For the purposes of this discussion, we will use the conception of tacit and explicit knowledge described by Nonaka (1994) in his seminal paper on knowledge creation34. Explicit knowledge is codified knowledge or information derived from knowledge. Tacit knowledge is personal knowledge; embodying elements like mental models, experience, skills, know-how and refers to individuals images of reality and visions for the future,(Nonaka 1994).

In the view of Boisot $(1998, \mathrm{p} 57)$ tacit knowledge comes in 3 distinct variants:

\footnotetext{
${ }^{3}$ Although this conception has gained some popularity in the literature, it should be emphasised that it conflicts with distinctions between tacit, explicit and implicit knowledge drawn by Polanyi $(1962 ; 1966)$ and subsequently adopted by Sveiby (1998), Baumard(1998), etc. We consider that there are some important consequences of these differences in conception and intend to address them in detail in a forthcoming paper.

${ }^{4}$ Burke(2000) provides a conceptually coherent discussion of knowledge in the context of competition and collaboration.
} 
- things that are not said because everybody understands them and takes them for granted

- things that are not said because nobody fully understands them. They remain elusive and inarticulate, see (Polanyi 1962))

- things that are not said because while some people understand them, they cannot costlessly articulate them, see Nonaka and Takeuchi (1995).

\section{Knowledge Creation}

Nonaka (1994, p 14) postulated that a stream of innovation indicates that an organisation should be studied with regard to how it creates information and knowledge, rather than how it processes problems. This insight suggests that we move away from the mechanistic model of organisations to seek a more dynamic, organic view.

Using the proposition that new knowledge is created through conversion between tacit and explicit knowledge Nonaka and Takeuchi (1995), have proposed that knowledge creation is a "spiralling process of interactions between explicit and tacit knowledge". Their SECI Model, shown, in Figure 2, suggests that there are four (dominant) knowledge conversion processes:

- Socialisation;

- Externalisation;

- Combination;

- Internalisation

Modes of Knowledge Creation

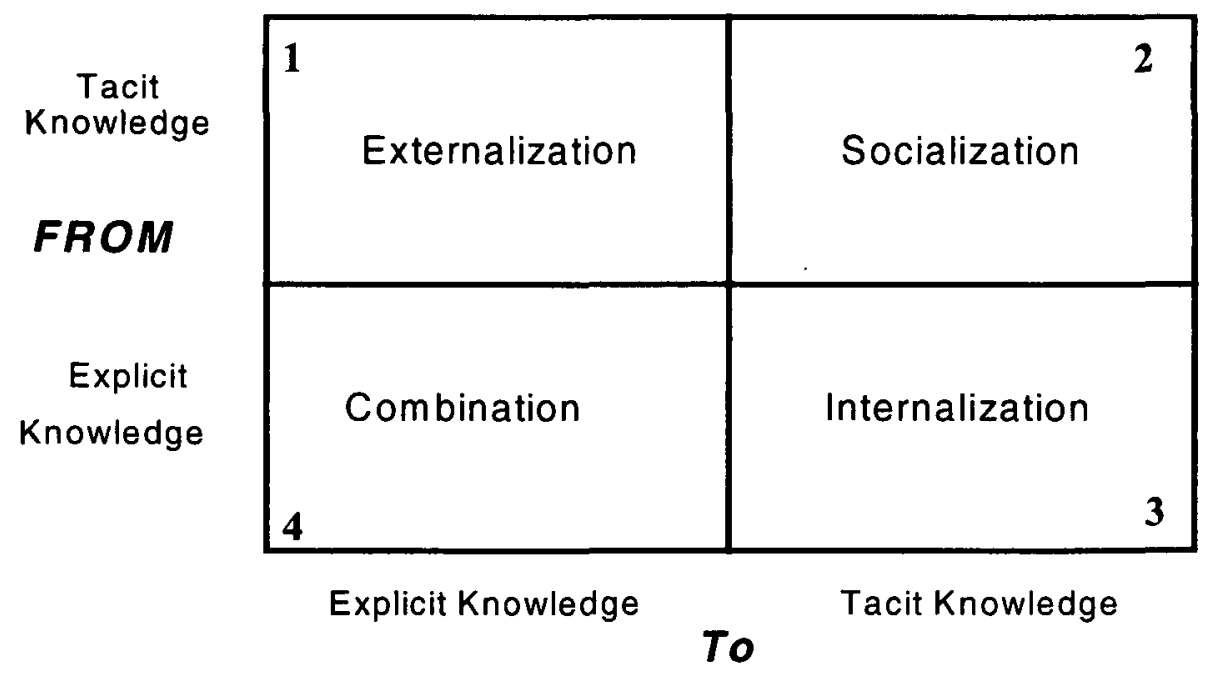

Figure 2. Modes of Knowledge Creation (Nonaka and Takeuchi 1995)

From studies of new product development in Japan, Nonaka (1994) found that the knowledge creation process needs a mix of creative chaos, redundancy of ideas, and requisite variety (towards the "crazy"). These conditions are quite different to those found in the efficient machine bureaucracy type of organisation (Handy 1986, p 192) that characterises Industrial Age production organisations and the traditional military structure (striving towards the "mild"). In the Knowledge Age, innovation is driving change at an increasing rate, which in turn is shaping changes in the organisational form. Miles et al (1997, $\mathrm{p} 7)$ describe how the organisational form has evolved as dominant competitive forces have changed. "The locus of organisational exemplars has shifted from capital intensive industries ... to information intensive industries, ... and now towards innovation-driven industries, where competitive advantage lies mostly in effective use of human resources".

\section{Information Systems}

The interplay between tacit and explicit knowledge provides a frame from which to look at the role of information technology in knowledge creation and Knowledge Management. Examination of Figure 2, shows that computers 
inherently operate in the lower lefi hand quadrant, where explicit codified knowledge is combined and manipulated into different and potentially more meaningful forms of explicit knowledge. The early stages of the Knowledge Age saw IS used to automate these processes, but as information and communications technology became more pervasive, new applications started to move into knowledge creation processes in the other quadrants. Web technology, for example, supports the learning process, the internalisation quadrant, by providing a mechanism for finding explicit knowledge that can be absorbed to add to an individual's tacit knowledge. Video-conferencing, groupware and meeting support software moves IT into the externalisation quadrant, and to some extent into the socialisation quadrant. These systems assist in making explicit some of the tacit knowledge held by those interacting with and through these systems.

\section{A SYSTEMS VIEW}

A systems view of the organisation is used to frame this study of Knowledge Management to establish a conceptually coherent basis for examining the issue. In this approach the organisation is viewed as a system operating within a wider system. The common conception of "system" within the disciplines of engineering and computer science is a physical, human-made, dynamic, combination of technologies, which acts in a coordinated way. Examples of such systems can range from desktop computers to submarines. However, natural and social scientists use the term to describe vastly different phenomena. Indeed, it was within the natural sciences that the term "system" was first popularised (von Bertalanffy 1968). Thus the term "system" is now used to describe things as diverse as ecological systems, human-activity systems, systems of ideas, and technical systems.

We contend that Systems Thinking is hierarchical and self-similar in nature. For example, the entire universe may be considered as one large system made from component galaxies, whilst the galaxies themselves can be viewed as systems of solar-systems, which in themselves are systems and so on and so forth. This reduction of abstraction can continue until we reach the level of the atom, which was once thought to be the smallest building block of the universe, but is now widely understood to be a system (of sub-atomic particles) as well. Such a structure of systems within systems can be thought of as a Systems Hierarchy5. Each system within the hierarchy is a "whole" with respect to its component elements, in addition to being a component element with respect to a system at a higher level in the hierarchy.

The art of thinking systemically about a part of the world is in the process of bounding both the object of study from its environment and the levels of abstraction (upper and lower) within the systems hierarchy. This process is described in detail in Matthews et al. (Matthews, Burke et al. 2000). For our purposes the process is important in that it brings into being the system of interest. The validity of Systems Thinking and analysis often depends on the appropriate choice of abstraction and an understanding of the mutual influences between the entity under study and the environment. Matthews et al. (Matthews, Burke et al. 2000) define five essential characteristics of a system (or Systems Thinking), these are component, component attributes, relationships, boundary and emergent properties (or system attributes). These characteristics are the recursive elements within the systems hierarchy, as shown in Figure 3.

A key issue in this conception of systems is that of emergence. An emergent property of a system is a property that is meaningful when attributed to the whole system, but not to its component elements (Checkland 1981). Thus it can be viewed as an attribute of the whole system that does not reside in any of the component elements6.

\footnotetext{
${ }^{5}$ A Systems Hierarchy is a self-similar structure; the pattem that recurs are is the five characteristics (described below) which make up the system concept.

${ }^{6}$ According to Capra (1996), the term "emergent properties" was coined by the philosopher C. D. Broad (1923), to refer to those system properties that emerge at a certain level (of hierarchy) but do not exist at lower levels. With this definition of emergence, a systems hierarchy can then be defined as an architecture view of a system from a structural perspective made on the basis of the existence of emergent properties. (Burke 2000).
} 


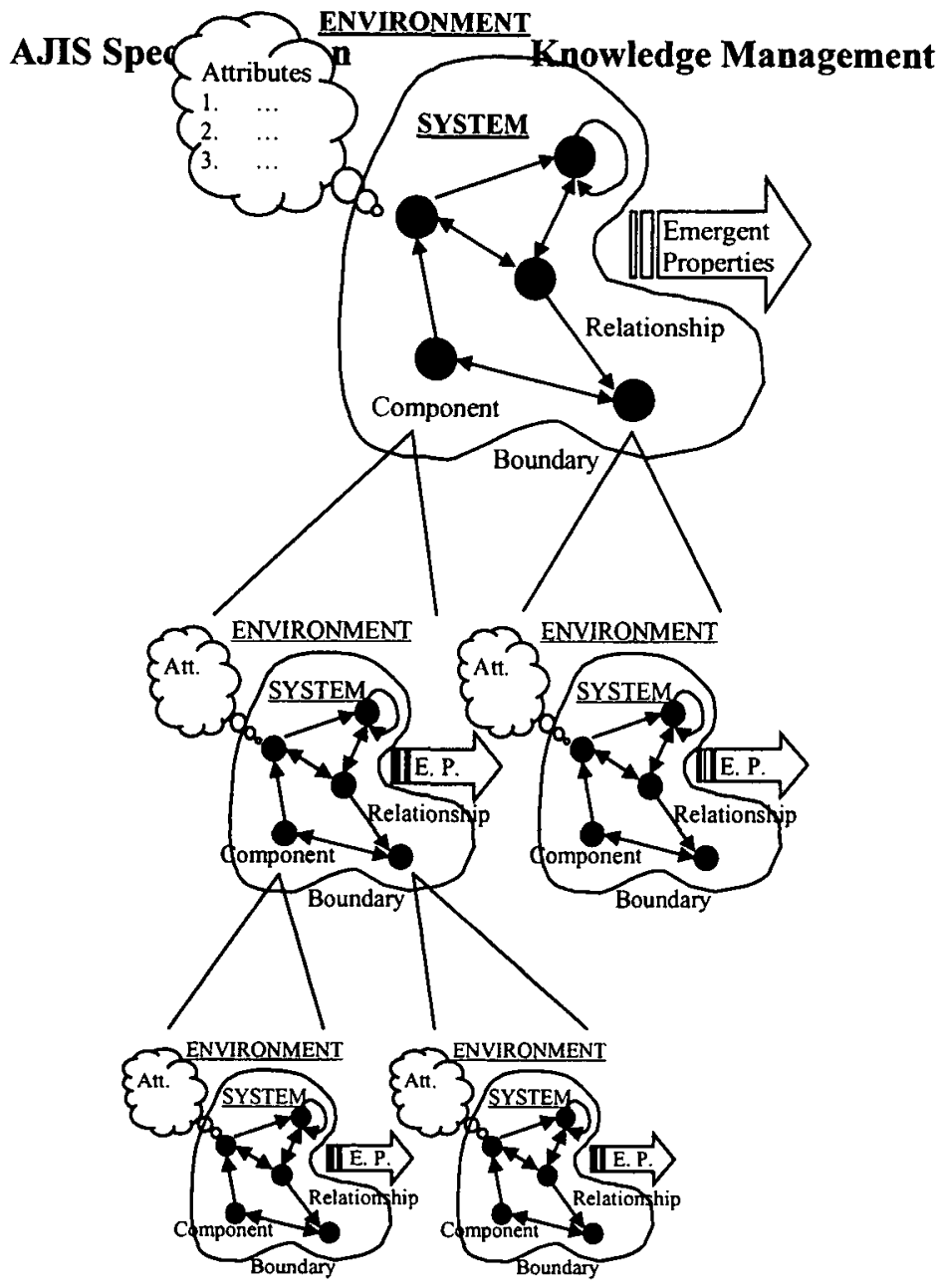

December 2001

Figure 3: A Repesentation of the Systems Hierarchy

\section{Organisations as Systems}

Organisations can be thought of as socio-technical systems composed of a complex interaction of humans and their tools. Kline defines socio-technical systems as "systems that link people with human-made hardware to perform tasks that humans want done" (Kline 1995, p. 171). Thus organisations are defined by the group of people in the organisation, the equipment they use and produce, as well as the procedures that govern the interaction of these components.

Another feature of the systems hierarchy is the recursive nature of the representation. Thus the emergent attributes of a system are the component attributes of the next highest system. Similarly, the relationships between the components within a system become the relationships with the environment when the component is the system of interest. The emergent attributes of the system under study is a property that emerges from the attributes of its components, the relationships among the components and the relationships between the systems and other systems in its environment.

These characteristics are evident in organisations, which inherently have components with attributes and relationships, and are seen to have boundaries and organisational properties (emergent attributes). An organisation's emergent system attributes include tangible products and services as well as less tangible attributes of organisational effectiveness, performance or strength. Components are organisational sub-groups in the organisation and the tools and procedures that are used in the organisation. Information Systems, for example, are an important socio-technical component of modern organisations, and their attributes and relationships with other parts of the organisation contribute to the emergent properties of the whole organisation. For many organisations in the Knowledge Age, Information Systems also mediate the external relationship that organisation has with other organisations in its environment.

It is emphasised that this simple description of an organisation is a representation of the organisation as a systems hierarchy from a limited perspective, namely that of organisational sub-groups, tools and procedures. From a 
broader perspective, organisations are made up of interlocking systems of components and relationships within an organisation. By selecting another viewpoint, different systems hierarchies can be seen within any organisation. Nonaka and Takeuchi, for example, describe this as the "Hypertext Organisation" (1995, p 169). Figure 4 shows one way of viewing components of an organisation with different relationships depending on which systems hierarchy view is taken. This leads to a richer, more complex view of the organisation as a system, compared to the "engineering" model of a system with components having well defined fixed relationships with each other, and producing predictable outputs. Thus a richer set of views provides a perspective that can generate insights into those components of an organisation operating in the "mild" the "wild" or the "crazy" regions.

Figure 4Multiple System Hierarchies in an Organisation

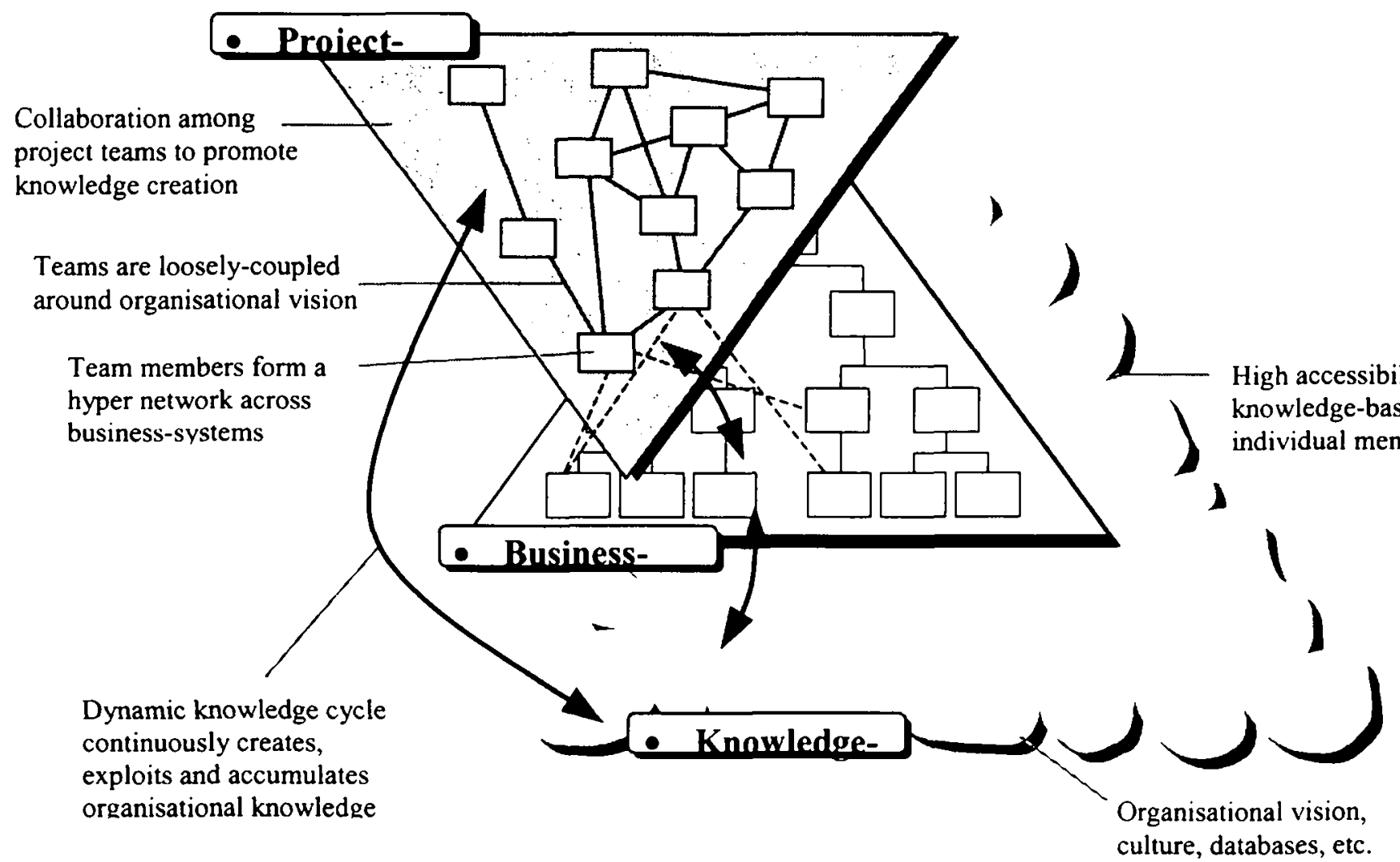

Similarly, (recursively) an organisation interacts in a complex way with its environment (the other components of the next highest system). This can be viewed from different systems hierarchy perspectives to gain further insights into the interactions. For simplicity, we will restrict discussion to a single view, using this as the archetype for other views. Acknowledging the potential for other views enables a more dynamic view of the organisation than is possible from a single view, particularly one drawn from a machine analogy viewpoint.

In recent times, the business and IS strategy literature has evolved to take account of the more dynamic environment (Stacey 1999; Hidding 2001). Models have evolved from structural forms (Porter 1980) to resource-based models (Prahalad and Hamel 1990; Barney 1991); moving from a reliance on predictions of the future environment, to developing capabilities that can be flexibly adapted to meet a range of contingencies.

\section{Effectiveness}

Effectiveness of the organisation can be viewed as the "fit" of its emergent attributes with those of its environment. How well system inputs are transformed into outputs to suit the environment determines the value of the organisation within the higher order system. Miles and Snow (1994) describe this as the process of "External Fit", while internal organisational effectiveness is linked with how well the components of the organisation achieve "Internal Fit".

"company success depends on putting together a complete and complementary package of ingredients: strategy, structure, process, and a managerial ideology that holds these together and gives them meaning. Further, this pack- 
age of characteristics must be widely understood and disseminated so that it becomes part of the daily behaviour of everyone in the organization. We call this process 'achieving fit"' (Miles and Snow 1994, p 7)

This description of what it takes to be a successful organisation emphasizes knowledge intensive issues of meaning, understanding and behaviour and "fits" with Kline's succinct definition of socio-technical systems as "systems that link people with human-made hardware to perform tasks that humans want done" (Kline 1995, p 171). An effective organisation then is a system that has a complementary set of components linked in such a way that the emergent properties of the system achieve desired goals.

\section{BALANCING NEAR THE EDGE}

To achieve internal "fit" the organisation has to achieve the complementary balance of components. Both the attributes of the component subsystems and their relationships can range from stable and predictable to volatile and unpredictable. In terms of the characteristics described in Figure 1, they include the mild, the wild, and the crazy.

The mild state represents stability, order and predictability, where well engineered components will have predicable behaviour. However, socio-technical systems can never achieve such certainty, because outcomes are not determined by fixed relationships, but emerge from a complex mix of rules, ethics, emotions and human drives interacting with each other and with human constructed tools. Nevertheless, systems can be designed and people trained and motivated to operate with a level of predictability and consistency that can tend towards the "edge of order".

Other socio-technical parts of the organisation are at the other end of the spectrum and (by design or accident) are out of control and unpredictable. These components of the organisation react faster and less predictably and are likely to drive the emergence of new and useful organisational attributes. At the same time, operating at the "edge of chaos" puts the organisation at risk of going over the edge into the "crazy" region of confusion, anarchy and disintegration.

Thus to be effective, an organisation needs components with attributes and relationships balanced in the "wild" band between stifling stability and potentially destructive chaos. Some organisations, for example, might at times operate in a stable environment while at other times have to cope with fluctuating market conditions. Like its components, the organisation itself operates in the "wild" striving to achieve an effective balance between the edge of order and the edge of chaos in its relationship with its environment.

Figure 5 shows a view of the interplay of the internal and external attributes and relationships, as they range between the edge of order and the edge of chaos. The mild and crazy states represent the limits of where we would expect to find components in an organisation or organisations in an environment.

Figure 5 Organisational Relationship - internal/external mix

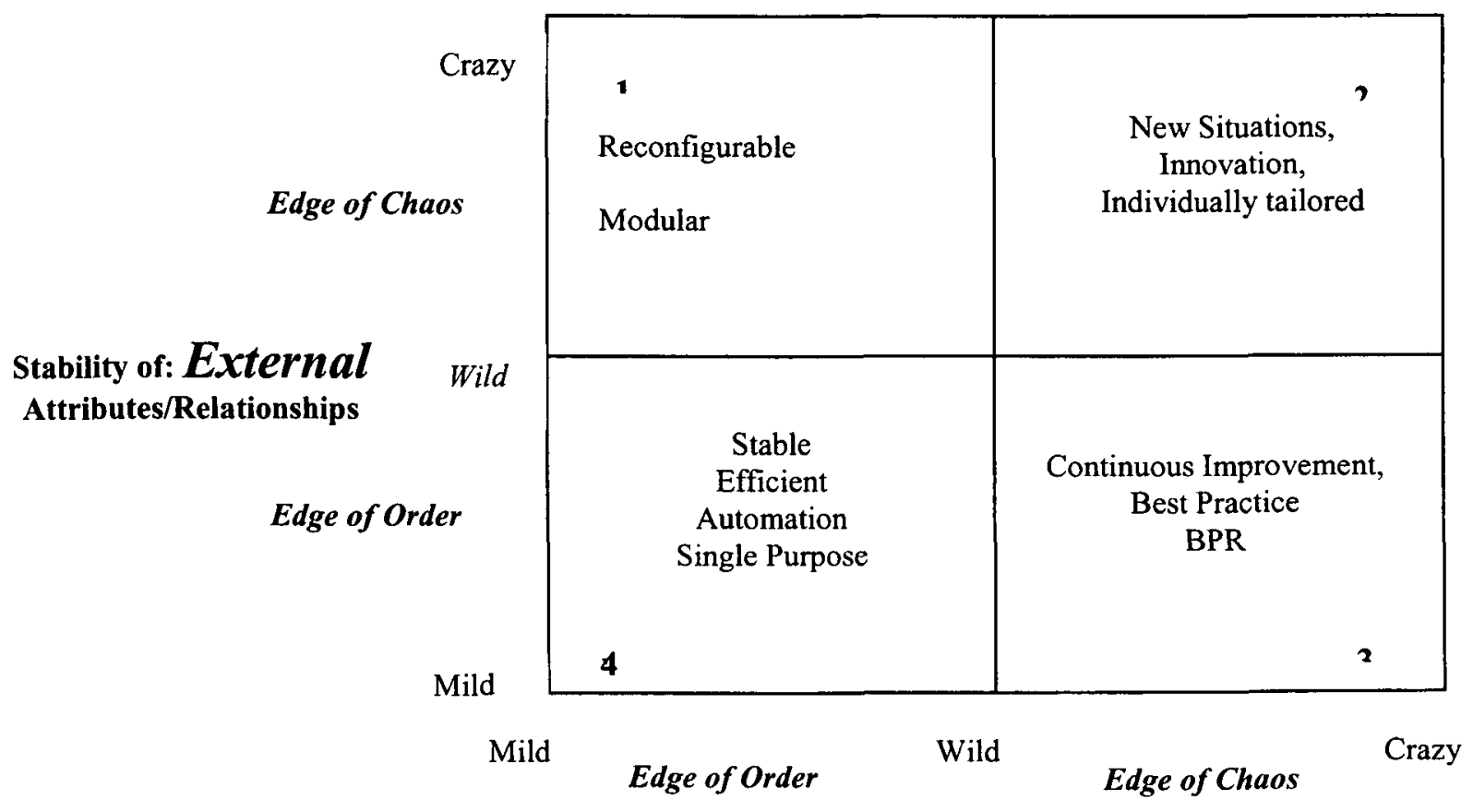

Stability of: Internal Attrib-

utes/Relationships 
This model 7 is of the organisation, but can be considered a general view of a typical layer of the systems hierarchy in which the sub-systems within, and the environment of, the organisation form a recurring self-similar structure. At the organisational level this model provides a tool to examine how its emergent properties in its environment relate to the relationships and attributes of its components.

For example, to be effective in quadrant 2 , the organisation, finding itself in an unstable rapidly changing environment, responds by moving itself towards the "crazy" to achieve the necessary innovation, adaptability and speed of response. However, such an organisation still needs components from quadrant 4 to provide stable infrastructure systems for the other components of the organisation. Lack of stable infrastructure can constrain effectiveness as much as an overly rigid and bureaucratic infrastructure. Thus, a quadrant 2 organisation will need components from all four quadrants, but in a mix that enhances organisational competitiveness.

As discussed above, knowledge plays a key role in the attributes and relationships within the organisation. In terms of the relationships described in Figure 5, the quadrant in which the organisation finds itself will be closely related to the predominant knowledge creation modes employed in the organisation.

\section{KNOWLEDGE MANAGEMENT STUDY OF THE AUSTRALIAN DEFENCE ORGANISATION}

\section{Background}

For 40 years following the end of World War II, the Cold War balance of the two world superpowers dominated military planning. In terms of our model, this external environment was near the "edge of order": it was predicable and stable. Accordingly, military forces could work to hone their internal processes to meet this threat efficiently. Inventions and technical developments, such as the developments in IT during this period, were incorporated to improve the efficiency of the huge Cold War "machines". During this time, the military world was solidly in quadrant 4 with inputs from quadrants 3 and 2 .

The collapse of the Soviet Union brought complex changes in the military environment: it swung from the mild to the crazy. Traditional approaches to military planning, organisation and practice were found wanting in this rapidly changing environment. New and unpredicted conflicts enupted, creating unexpected alliances and unstable coalitions. Military forces, structured for large-scale conflict against similar forces, had to be quickly adapted to handle asymmetric conflict. The attributes and relationships optimised over the years to be effective in the stable, balanced force world were now inappropriate to cope effectively with the changing attributes and relationships of military conflict. The military world was now in quadrant 2 , with military planners having to cobble together quickly one-off solutions for unexpected situations.

The Australian Defence Organisation (ADO), like its counterparts across the world, has been working hard to understand and adapt to the unsettling changes in the military landscape since the end of the Cold War. Also, like all organisations, the ADO continues to evolve its practices and processes to make best use of developments in Information Systems. One response of the ADO to these pressures was to appoint, in mid 2000, its first Chief Knowledge Officer who commissioned a team from the Defence Science \& Technology Organisation (DSTO) to investigate Knowledge Management in the Defence Headquarters. Aspects of the findings from this study motivated much of the thinking behind this paper.

\section{Method}

During the study the team interviewed 60 staff comprising a broad cross section from the ADO headquarters located in Russell, in the Australian Capital Territory. Interviewees were selected for their interest in KM and came from the three single services and the civilian staff. The rank of those interviewed ranged from Chief of the Defence Force and Secretary of the Department to trainee Graduate Administrative Assistants, but centred around the "one star" (Brigadier and equivalent) level. Initially, the team planned to conduct structured interviews, but moved to a more open format in order to capture the rich range of experience, opinions and ideas from the staff interviewed. Although this adaptation was expedient in achieving CKO's immediate goals in commissioning the study, it has consequences for the scientific rigour of the approach and consequently its findings need to be interpreted accordingly. To make sense of the wealth of information contained in the interview notes, the team aggregated the interviewee comments into various themes and groupings. General themes emerged around issues of Culture, Organisation, Learning and Technology. The comments in these groups were further divided into comments on KM enablers, inhibitors, initiatives and recommendations. This analysis provided the CKO with a structured snapshot of perceptions

\footnotetext{
${ }^{7}$ This model also builds on Pine's (1993) "Mass Customization" model of Product/Process change in manufacturing.
} 
of $\mathrm{KM}$ within the Canberra headquarters. It also gave the study team a valuable perspective of KM issues from a key part of the Australian Defence Organisation that, amongst other things, provoked the thinking leading to the development of the model presented above.

\section{Selected observations}

Many of those with whom we spoke had formed clear ideas on how to best to improve Knowledge Management, but no clear consensus emerged. In fact the perceptions and recommendations, on both cultural and technology issues, seemed to be in conflict.

It is emphasised that the observations reproduced below have been selected by the authors to illustrate the contrasting ideas. They are (often strongly worded) opinions of individuals on particular issues of interest to them. It is important that they should not be interpreted as representing: the formal position of the ADO; a complete and coherent description of $\mathrm{KM}$ in $\mathrm{ADO}$; or the consensus view of those interviewed.

Those interviewed believed that the culture of the organisation was central to improving Knowledge Management in the $\mathrm{ADO}$, but voiced conflicting requirements. Some, who perceived a requirement for the organisation to be able to survive in an environment of rapid change, identified the need for more flexibility and even chaos, to promote a culture in which innovation and flexibility could flourish. Others, who understood the need for strong risk management in a business with as much at stake as defence of the country, identified the need for a more structured, highreliability culture.

Some, who advocated a more open culture, saw promise in the attitudes of those entering the service: 'Young people are more open in their interactions with senior officers', but others believed that the military culture could potentially constrain the innovation and flexibility needed to be effective in a changing environment. A civilian offered the opinion that Defence is: 'Not open, not sharing'.

On the other hand, others saw more structure and control as the key to improved $\mathrm{KM}$ and organisational effectiveness. Particular concerns were held in regard to records control and management that were seen as necessary to restore efficiency, reduce duplication and retain corporate knowledge. One officer commented on the need to facilitate cultural change to get people to understand that reporting is part of business'. 'Compliance with the Archives Act is not optional'.

The responses in relation to the Information Systems showed the same paradox. Some advocating relaxing some controls on IS, while others saw tighter controls as the way to improve organisational effectiveness. Ideas for opening up the IS constraints included improving linkages with outside agencies and more freedom to introduce or experiment with new tools and databases. As it was put succinctly by an interviewee, there is 'No provision of technology to support sharing, DefWeb8 is not searchable, cannot see outside branches.' Another military officer related his experience with setting up a chat room tool, which 'was closed down unilaterally by CISMO' (the Central IS Management Organisation).

The contrary view was put by those who believed improved KM would come from tighter procedures to control document storage and sharing, standardisation of tools to enable sharing across the organisation, as well as tighter controls to improve system reliability and reduce the risk of unauthorised access to information. One officer suggested 'The Configuration Control Board in DIEB is the way to enforce compliance'. Another could see the need for standards, as a pre-requisite for establishing $\mathrm{KM}$. To quote, there is a 'Need to define standards and procedures: e.g. common Web environment and images. Develop systems across board'.

\section{Discussion}

These informal comments do not constitute fully rigorous scientific findings 9 , but do highlight an interesting question. How is it possible for concerned, dedicated and intelligent people, who have all thought deeply about Knowledge Management in their organisation, to come to such divergent opinions on the best way forward?

The model in Figure 5 provides insights into this paradox. During the Cold War there was a relatively stable military strategic environment in which the external attributes and relationships of the ADO evolved slowly over time, but within a known superpower framework. Internal attributes and relationships in the ADO also evolved and adapted in a relatively stable way, with changes in technology and organisation focussed on more efficient operation within the predictable external framework. Many of the people interviewed had received their training and much of their expe-

\footnotetext{
${ }^{8}$ DefWeb is the ADO intranet.

${ }^{9}$ They do, however, constitute a preliminary survey that would provide a useful basis for a more systematic, scientific investigation. Work of this nature may be undertaken at some stage in the future.
} 
rience in this era and some had come to see a world with a predictable framework and efficient internal processes, as "how things should be". This situation is identified with quadrant 4 , where the rate of change of the external attributes and relationships is "mild" and internal characteristics also have an orderly evolution. Both external and internal relationships are close to the "edge of order". In this mode, streamlining the organisation is represented by excursions into quadrant 3 to improve internal processes, before retuming to quadrant 4 with improved efficiency to meet the known external environment. Coping with a disruptive new invention or event was achieved by stabilising a quadrant 2 development in such a way that it could be incorporated back into the quadrant 4 world.

With the dramatic change in the strategic military situation in recent times, external relationships have become wildly unpredictable, balancing on the "edge of chaos". Internal attributes and relationships, developed in response to the Cold War influences, have consequently become unstable. What was best practice in the Cold War era becomes inappropriate or inefficient when meeting emerging situations. Here the ADO is operating in quadrant 2 . The INTERFET peacekeeping mission was an example of this. New and different relationships had to be created very quickly. With Australia leading the coalition, the relationship with the USA was nothing like that honed to a high state of efficiency during the Cold War. Simultaneously, working relationships within a diverse 22 nation coalition had to be developed. Also, although the command arrangements within the Australian Defence Force had already undergone significant change to accommodate the post Cold War situation, they had to be changed quickly and adapted to meet this unpredicted INTERFET situation.

It seems inevitable from recent events that external attributes and relationships for the ADO are likely to remain on the edge of chaos. The ADO would be more effective if it could move from quadrant 2 to operate in quadrant 1 . To operate in this mode, stable internal attributes and relationships need to be developed in such a way that they can be reconfigured flexibly to accommodate unexpected requirements. The problem becomes one of determining which attributes, relationships and processes should be stabilised to form the efficient backbone on which capabilities can be efficiently mixed and matched to meet sudden changes in the external environment. Striving to operate in this quadrant is what, we believe, led to the apparently contradictory comments from those interviewed in the Knowledge Management Study.

Those interviewees who commented on the need for more flexibility were identifying the need for organisation relationships and attributes that could emerge quickly to accommodate the extemal environment effectively. Those who sought more control and stability were focussed on the need to develop an organisation with a strong and stable framework that could deal efficiently with the extemal environment. Within this framework, the planning issue for this organisation comes down to devising processes, attributes and relationships that can be managed tightly to provide efficiency, without restricting the flexibility needed to quickly adapt to unpredictable changes to the external systems. In terms of the KM study, this is the recognition of the need for underlying modules of technology and associated operating procedures operating efficiently "on the edge of order", but with characteristics that allow for rapid reconfiguration to interact effectively with whatever attributes emerged to change relationships with the external environment. In summary, the $\mathrm{ADO}$ is a quadrant 4 organisation that has been thrust into quadrant 2 , and is moving to achieve greater organisational effectiveness by attempting to move to quadrant 1 .

\section{IMPLICATIONS FOR KNOWLEDGE MANAGEMENT AND SYSTEMS THINKING}

\section{Knowledge Management}

The Knowledge Management debate has tended to be dominated by the software and Information Systems issues, which has distorted the approach to the management of knowledge in an organisation. Berkman (2001), for example, sees this a consequence of the marketing activities of software vendors. As discussed above, Knowledge Management, particularly when seen as a knowledge creation issue, involves dynamic interplay between tacit and explicit knowledge. KM needs to include cultural as well as technological elements. The risk of not mobilising knowledge in the organisation is compounded if Knowledge Management is perceived as a requirement that can be met with a traditionally engineered software solution. A simplistic software approach to Knowledge Management is likely to not only leave out the management of tacit knowledge, but is also prone to focus on solving a particular issue, rather than developing a capability.

\section{Systems Thinking}

The Systems Thinking concept used in this paper is a useful structure within which to frame the problem of balancing the relationships among the "mild, wild, and crazy" components of an organisation. The self-similar concept of the hierarchy simplified the understanding of the emergent attributes and the relationships at each level. The model 
described above can be applied recursively to the organisation and its environment and down through the layers of systems within the organisation. This model can be used as an explanatory tool, and as a framework for developing future strategy for an organisation and the subsystems within it.

Defining the upper and lower boundaries of the organisation, its components and environment is a difficult issue. The upper boundary is that which defines the system of interest, in our case the organisation. The upper boundary of a government department, for example, might seem to be well defined, but aspects of outsourcing and consultants, for example, cloud the boundary definition. Similarly, the lower boundary, which is that which defines the separately identifiable "atomic" components of the system (organisation), is difficult to define exactly. Invariably, such boundaries change over time and also differ within alternate system views of the organisation, such as those in Figure 4.

Further work needs to done to extend the model to take account of the other systems hierarchies that simultaneously exist within the organisation. This was briefly discussed in relation to Figure 4 that shows diagrammatically two of the many structures that exist in an organisation. Useful insights come from considering the single hierarchy, but in many cases the cross linking (termed 'hypertext organisation' by Nonaka and Takeuchi) reveal other emergent attributes of the components of the organisation and of the organisation itself. Novel approaches (e.g. Shaw 1997), beyond those of established system analysis techniques, will need to be developed if we are to tap into this richer systems understanding of the organisation.

\section{CONCLUSION}

As the world moves into the Knowledge Age, the rate of change of the environment in which organisations operate is developing at an ever-increasing pace driven, in large measure, by growth in the capabilities of information and communications technology. With issues of knowledge and innovation now critical to performance in many organisations, traditional approaches to improving organisational performance, based on concepts of organisations as mechanistic or "Newtonian" systems have been found wanting. This paper draws together concepts from Complexity Science, Systems Thinking and Knowledge Management to develop a model relating the effectiveness of an organisation to the complexity of its internal and external attributes and relationships. Despite its simplicity, the model proved to be a useful lens for observing the Defence Organisation: at a coarse level of resolution it clarified important differences in the perception of Knowledge Management in Defence. We believe that it might be similarly useful in analysis of other organisations. Further work is needed to improve understanding of how the "mild, wild, and crazy" component parts interact within organisations.

\section{REFERENCES}

Barney, J. B. (1991). "Firm resources and sustained competitive advantage." Journal of Management Vol. 17, No. 1: pp. 99-120.

Baumard, P. (1998). "From InfoWar to Knowledge Warfare: Preparing for the Paradigm Shift", http://www.iae.univaix.fr/cv/baumard/infowar knowledge warfare.htm. Accessed: 2 Nov 2001.

Berkman, E. (2001). "When bad things happen to good ideas-Knowledge Management", http://www.darwinmag.com/read/040101/badthings.html. Accessed: 14 June 2001.

Boisot, M. H. (1998). Knowledge assets: securing competitive advantage in the information economy. Oxford, Oxford University Press.

Broad, C. D. (1923). The mind and its place in nature. London, Kegan Paul.

Burke, M. (2000). "Thinking Together: New Forms of Thought System for a Revolution in Military Affairs", Defence Science and Technology Organisation.

Capra, F. (1996). The Web of Life: a new synthesis of life and matter. London, Flamingo.

Checkland, P. (1981). Systems Thinking, Systems Practice. Chichester, John Wiley \& Sons.

Freedman, D. H. (1992). “Is Management Still a Science?" Harvard Business Review(Nov-Dec1992): pp. 26-38.

Gleik, J. (1988). Chaos. London, William Heinemann.

Handy, C. B. (1986). Understanding Organisations. Hammondsworth, England, Penguin Books.

Hidding, G. J. (2001). "Sustaining strategic IT advantage in the information age: how strategy paradigms differ by speed." Journal of Strategic Information Systems 10(3): 201-222.

Kline, S. J. (1995). Conceptual Foundations for Multidisciplinary Thinking. Stanford, Stanford University Press.

Kuhn, T. S. (1970). The Structure of Scientific Revolutions. Chicago, The University of Chicago Press.

Matthews, D., M. Burke, et al. (2000). "Core Concepts of Joint Systems". Australasian Systems Engineering, Test and Evaluation Conference, Brisbane. 
Matthews, D. B., M. Burke, et al. (2000). "Assessing the value of a Joint Systems capability". Australasian Systems Engineering, Test and Evaluation Conference, Brisbane.

Miles, R. E. and C. C. Snow (1994). Fit, Failure and the Hall of Fame: how companies succeed or fail. New York, The Free Press.

Miles, R. E., C. C. Snow, et al. (1997). "Organising in the Knowledge Age: Anticipating the cellular form." Academy of Management Executive Vol. 11 No 4: pp 7-20.

Mintzberg, H. (1994). The rise and fall of strategic planning: reconceiving roles for planning, plans, planners. New York, The Free Press.

Nonaka, I. (1994). "A Dynamic Theory of Organizational Knowledge Creation." Organization Science Vol. 5, No. 1(Feb 1994): 14-37.

Nonaka, I. and H. Takeuchi (1995). The Knowledge-Creating Company. New York, Oxford University Press.

Pine II, B. J. (1993). Mass Customization: the new frontier in business competition. Boston, Harvard Business School Press.

Polanyi, M. (1962). Personal Knowledge : towards a post-critical philosophy. Chicago, University of Chicago Press.

Polanyi, M. (1966). The Tacit Dimension. London, Routledge \& Kegan.

Porter, M. E. (1980). Competitive strategy: techniques for analysing industries and competitors. New York, Free Press.

Prahalad, C. K. and G. Hamel (1990). "The core competence of the corporation.” Harvard Business Review(MayJune 1990): 79-91

Pugh, D. S. and D. J. Hickson (1996). Writers on organisations. London, Penguin Books.

Rebello, K. (1996). "Inside Microsoft : The untold story of how the Internet forced Bill Gates to reverse course", http://www.businessweek.com/1996/b34841.htm. Accessed: 16 December 1997.

Sauer, C. and P. W. Yetton, Eds. (1997). Steps to the Future: Fresh thinking on the management of IT-based organisational transformation. San Francisco, Jossey-Bass.

Shaw, P. (1997). "Intervening in the shadow systems of organizations Consulting from a complexity perspective." Journal of Organizational Change Management 10(3): 235-250.

Stacey, R. D. (1996). Complexity and creativity in organizations. San Francisco, CA, Berrett-Koehler.

Stacey, R. D. (1999). Strategic management and organisational dynamics : the challenge of complexity. New York, Financial Times.

Sveiby, K. E. (1998). "Tacit Knowledge, Sveiby Knowledge Management", http://www.sveiby.com.au/Polanyi.html. Accessed: 15 Nov 2001.

Taylor, F. W. (1923). The Principles of Scientific Management. New York, Harper and Brothers.

The Price Waterhouse LLP Change Integration Team (1996). The paradox principles : how high-performance companies manage chaos, complexity, and contradiction to achieve superior results. Chicago, Ill., Irwin Professional.

Turner, D. and M. Crawford (1998). Change Power: capabilities that drive corporate renewal. Warriewood, NSW, Business and Professional Publishing.

von Bertalanffy, L. (1968). General Systems Theory: foundations, development, applications. New York, George Braziller. 